\title{
Synthesis Method and Experimental Research of Ku-band Antennas Based on Tensorial Metasurfaces
}

\author{
Konstantin V. Lemberg*a, \\ Eugeny O. Grushevsky ${ }^{a}$, Ivan V. Podshivalov', \\ Alexey N. Kosmynin ${ }^{b}$ and Ksenia N. Kosmyninab \\ ${ }^{a}$ Kirensky Institute of Physics SB RAS \\ Krasnoyarsk, Russian Federation \\ ${ }^{b}$ Matrix Wave LLC \\ Moscow, Russian Federation
}

Received 01.09.2021, received in revised form 15.10.2021, accepted 20.10.2021

\begin{abstract}
Antennas based on metasurfaces with tensorial impedance is a relatively new type of antennas appeared in the last decade. They have a simple design, but a complex synthesis procedure. The synthesis procedure can be based on analysis of the properties of waves in periodic structures or on representing the antenna as a phase hologram. Due to the novelty of this type of antennas, researching of various synthesis methods, obtaining new experimental results and on their basis assessment of realistically achievable characteristics is of interest. The purpose of the work was an experimental research of antennas based on metasurfaces with tensorial surface impedance, synthesized according to the method proposed by the authors. To carry out the research, mathematical modeling, electrodynamic modeling, and nearfield measurements of antenna prototypes were used. As a result, it was experimentally confirmed that the proposed tensorial metasurface antennas synthesis method makes it possible to obtain antennas with a given radiation direction and polarization, which have an aperture efficiency of up to $50 \%$, a bandwidth of up to $8 \%$, and a cross-polarization level below minus $20 \mathrm{~dB}$. The results of measurements are in a good agreement with the electrodynamic calculation and the parameters specified during the synthesis. The type of antennas described in the work and the given synthesis method, according to the authors, are promising for various applications, for example, in satellite and mobile communications.
\end{abstract}

Keywords: metasurface, metasurface antenna, leaky waves, surface impedance, tensorial impedance, holographic beamforming, Ku-band.

Citation: Lemberg K. V., Grushevsky E. O., Podshivalov I. V., Kosmynin A. N., Kosmynina K. N. Synthesis method and experimental research of ku-band antennas based on tensorial metasurfaces, J. Sib. Fed. Univ. Eng. \& Technol., 2021, 14(7), 840-853. DOI: 10.17516/1999-494X-0358

(c) Siberian Federal University. All rights reserved

This work is licensed under a Creative Commons Attribution-Non Commercial 4.0 International License (CC BY-NC 4.0).

* Corresponding author E-mail address: lemkon@inbox.ru 


\title{
Методика синтеза и экспериментальное исследование
}

\section{антенн Кu-диапазона}

на основе тензорных метаповерхностей

\author{
К. В. Лемберга \\ И.В. Подшивалов ${ }^{\text {, }, ~ А . Н . ~ К о с м ы н и н ~}{ }^{\sigma}$, К.Н. Космынина \\ ${ }^{a}$ Институт физики им. Л. В. Киренского ФИЦ КНЦ СО РАН \\ Российская Федераџия, Красноярск \\ ${ }^{\sigma} О О О$ «Мэтрикс Вейв» \\ Российская Федерация, Москва
}

\begin{abstract}
Аннотация. Антенны на основе тензорных метаповерхностей - это сравнительно новый тип антенн, появившийся в последнее десятилетие. Они отличаются простой конструкцией, но сложной процедурой синтеза, которая может быть основана на анализе свойств волн в периодических структурах или на основе интерпретации антенны как фазовой голограммы. В связи с новизной этого типа антенн представляет интерес исследование различных методик их синтеза, получение новых экспериментальных результатов и оценка на их основе реально достижимых характеристик таких антенн. Целью работы являлось экспериментальное исследование антенн на основе тензорных метаповерхностей с модулированным поверхностным импедансом, синтезированных по предложенной авторами методике. Для проведения исследований было использовано математическое моделирование, электродинамическое моделирование и измерения макетов антенн в ближней зоне излучения.

В результате работы экспериментально подтверждено, что предложенная методика синтеза антенн на основе тензорных метаповерхностей позволяет получать антенны с заданными направлением излучения и поляризацией, которые обладают апертурной эффективностью до 50 \%, шириной полосы рабочих частот до 8 \% и уровнем кросс-поляризации ниже минус 20 дБ. Результаты измерений антенн хорошо согласуются с электродинамическим расчетом и заданными при синтезе параметрами. Описанный в работе тип антенн и приведенная методика их синтеза перспективны для ряда применений, например в спутниковой и мобильной связи.
\end{abstract}

Ключевые слова: метаповерхность, антенна на основе метаповерхности, вытекающие волны, поверхностный импеданс, тензорный импеданс, голографическое диаграммообразование, Kuдиапазон.

Цитирование: Лемберг, К. В. Методика синтеза и экспериментальное исследование антенн Ки-диапазона на основе тензорных метаповерхностей / К. В. Лемберг, Е. О. Грушевский, И. В. Подшивалов, А. Н. Космынин, К. Н. Космынина // Журн. Сиб. федер. ун-та. Техника и технологии, 2021, 14(7). C. 840-853. DOI: 10.17516/1999-494X-0358

Введение. Метаповерхностями называют двумерные периодические структуры с периодом много меньше длины волны, электромагнитные свойства которых определяются искусственно созданными субволновыми элементами. В последнее десятилетие метаповерхности находят все больше практических применений в СВЧ-технике [1]. Одним из таких применений являются антенны на основе метаповерхностей с модулированным поверхностным импедансом (далее АМП). АМП относятся к так называемым антеннам «вытекающей» волны [2], физический принцип работы которых заключается в излучении быстрой (т. е. имеющей фазовую скорость больше скорости света) волны из открытой волноведущей структуры. Поверхностная волна ТМ-типа, используемая в АМП, медленная (т. е. имеет фазовую скорость меньше скорости света), однако

$$
-841-
$$


для излучения электромагнитных волн используется быстрая минус первая пространственная гармоника, образующаяся благодаря периодической модуляции поверхностного импеданса используемой двумерной волноведущей структуры. В качестве такой волноведущей структуры в сантиметровом диапазоне длин волн может применяться диэлектрическая подложка, экранированная с нижней стороны и имеющая субволновый рисунок металлизации (метаповерхность) с верхней стороны. Применение метаповерхностей для создания антенн вытекающей волны позволяет существенно более гибко, чем в других антеннах этого класса, управлять излучением электромагнитных волн.

Анализ электромагнитных структур с импедансными поверхностями для различных применений проводился [3-5] задолго до появления термина «метаповерхность». Однако эти структуры имели скалярный импеданс, а их синтез проводился аналитически. Антенны с тензорным поверхностным импедансом требуют при синтезе значительного объема численных расчетов, в связи с чем активное развитие это направление получило с начала 2010-х годов [6-13]. Было показано, что АМП с тензорным импедансом могут обладать высокой апертурной эффективностью и формировать диаграммы направленности специальной формы.

Отличительными особенностями АМП являются, с одной стороны, простота конструкции (антенна в сантиметровом диапазоне длин волн может быть выполнена в виде однослойной печатной платы), с другой - достаточно сложный метод синтеза. В некоторых работах антенны на основе метаповерхностей называют антеннами с голографическим диаграммообразованием $[14,15]$, что связано с тем, что они могут быть рассмотрены как фазовые голограммы, преобразующие опорную волну (поверхностную волну ТМ-типа) в объектную волну (ТЕМ-волну, излучаемую в свободное пространство). В связи с этим методы синтеза АМП можно разделить на два класса: использующие подход на основе анализа волн в периодических волноведущих структурах и использующие голографический подход.

В данной работе описан принцип работы антенн на основе метаповерхностей с модулированным поверхностным импедансом, приводится разработанная авторами методика синтеза антенн на основе тензорных метаповерхностей, основанная на голографическом подходе, а также результаты экспериментального исследования трех АМП Ки-диапазона.

Принцип работы антенн на основе модулированных метаповерхностей. Как известно, волны в волноведущих структурах по соотношению фазовой скорости $v_{p h}$ и скорости света $c$ могут быть разделены на медленные $\left(v_{p h}<c\right)$ и быстрые $\left(v_{p h}>c\right)$. При распространении волны вдоль неэкранированной поверхности без потерь (рис. 1) ее волновое число $\beta$ будет иметь значение меньше или больше волнового числа волны в свободном пространстве $k_{0}$, для медленных и быстрых волн соответственно. При этом значения $\beta$ и $k_{0}$ связаны соотношением

$$
k_{0}^{2}=\beta^{2}+k_{z}^{2}
$$

где $k_{z}$ - волновое число в направлении, перпендикулярном направлению распространения. Так как

$$
k_{0}=\frac{2 \pi}{\lambda_{0}}
$$

где $\lambda_{0}$ - длина волны в свободном пространстве, $k_{z}$ будет действительным или мнимым для быстрых и медленных волн соответственно. 


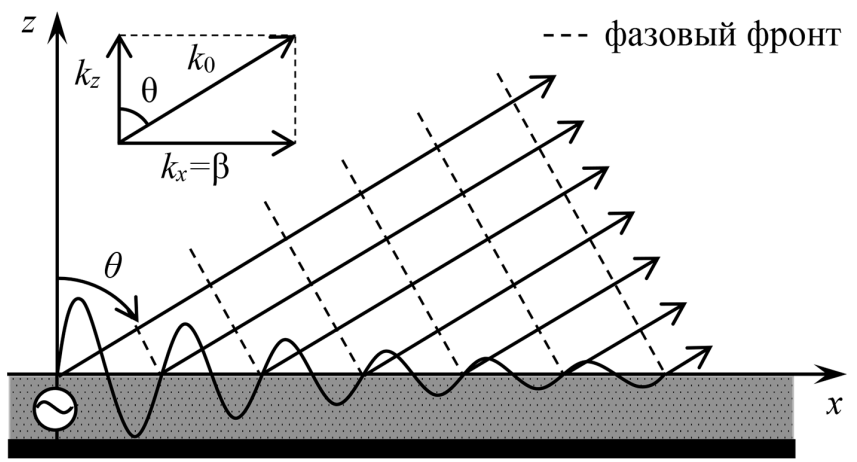

Рис. 1. Механизм излучения быстрых волн в неэкранированной волноведущей структуре

Fig. 1. Fast waves radiation principle in non-shielded waveguiding structure

Если $\beta>k_{0}\left(v_{p h}<c\right)$, то $k_{z}$ оказывается мнимой величиной, т. е. излучения волны не происходит, а поле волны экспоненциально убывает при удалении от поверхности. В этом случае волна поверхностная, т. е. является как бы «прижатой» к поверхности и распространяется вдоль нее без излучения.

Если $\beta>k_{0}\left(v_{p h}>c\right)$, то значение $k_{z}$ оказывается действительным, и происходит излучение под углом $\theta$ от нормали к поверхности таким, что (см. рис. 1)

$$
\sin \theta=\frac{\beta}{k_{0}}=\frac{c}{v_{p h}} .
$$

Таким образом, в этом случае волновой процесс не «привязан» к поверхности и по мере распространения волны происходит ее излучение в свободное пространство. Такую волну называют вытекающей. Излучение за счет вытекающих волн может иметь место как для одномерных, так и для двумерных волноведущих структур.

В АМП используется механизм излучения медленных поверхностных волн, который имеет место, если импеданс волноведущей структуры, вдоль которой распространяется волна, периодически изменяется. Как известно, волны в периодических структурах могут быть представлены в виде суммы пространственных гармоник (гармоник Флоке), которые на дисперсионной диаграмме расположены с периодом $2 \pi / p$, где $p$ - период структуры. На этой диаграмме (рис. 2) быстрые волны располагаются внутри светового конуса $\omega \beta= \pm c$, а медленные - вне его.

В случае синусоидальной модуляции импеданса появляются, кроме основной (нулевой, $n=0)$ пространственной гармоники, соответствующей полю волны в немодулированной структуре, еще две пространственные гармоники - плюс первая $(n=+1)$ и минус первая $(n=-1)$. Минус первая пространственная гармоника имеет постоянную распространения

$$
\beta_{(-1)}=\beta_{(0)}-\frac{2 \pi}{p}
$$

и, соответственно, фазовую скорость

$$
v_{p h(-1)}=\frac{\omega}{\beta_{(-1)}}=\frac{\omega}{\beta_{(0)}-\frac{2 \pi}{p}} .
$$




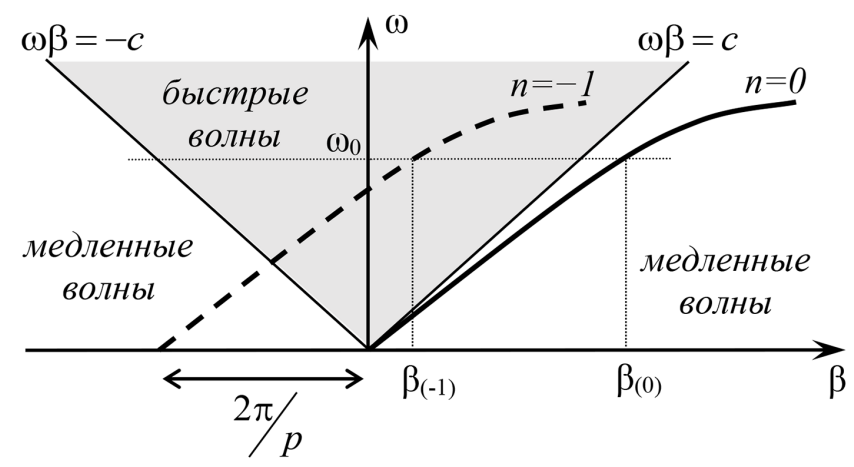

Рис. 2. Дисперсионная диаграмма медленной волны для случая синусоидальной модуляции импеданса поверхности

Fig. 2. Slow wave dispersion diagram in the case of sinusoidal surface impedance modulation

Если период модуляции подобран так, что на рабочей частоте $v_{p h(-1)}>c$, то поверхностная волна будет излучаться под углом, определяемым формулой (1). При этом нулевая и минус первая пространственные гармоники являются частью одного волнового процесса, поэтому излучение в свободное пространство происходит за счет энергии волны в целом. Интенсивность излучения определяется амплитудой минус первой пространственной гармоники, которая зависит от глубины модуляции импеданса волноведущей структуры

$$
m=\frac{\Delta Z}{\bar{Z}},
$$

где $\bar{Z}$ - среднее значение импеданса, а $\Delta Z$ - диапазон его изменения. В отсутствие тепловых потерь этот импеданс является чисто мнимым. При этом для двумерной волноведущей структуры (поверхности) импеданс может быть тензорным, т. е. описываться тензором вида

$$
\hat{Z}_{s}=\left[\begin{array}{ll}
Z_{u u} & Z_{u v} \\
Z_{v u} & Z_{v v}
\end{array}\right],
$$

где $u, v$ - орты выбранной на поверхности системы координат. Тензор поверхностного импеданса связывает тангенциальные компоненты напряженности электрического $E_{t}$ и магнитного $H_{t}$ полей на поверхности:

$$
E_{t}=\hat{Z}_{s} \times\left[\boldsymbol{n} \times H_{t}\right]
$$

где $\boldsymbol{n}$ - единичный вектор, направленный по нормали к поверхности. Каждый компонент тензора может иметь собственный закон модуляции. Тензорный характер импеданса позволяет связывать ортогональные компоненты полей на поверхности, благодаря чему появляется возможность управления поляризационными характеристиками излучения.

Таким образом, с помощью модуляции тензорного импеданса поверхности можно управлять направлением, интенсивностью и поляризацией излучения медленных поверхностных волн.

Методика синтеза антенны. Физическая реализация метаповерхностей с тензорным поверхностным импедансом может быть различной. Например, в миллиметровом диапа- 
зоне длин волн они могут быть выполнены в виде металлической структуры, изготовленной с помощью 3D-печати [16]. В сантиметровом же диапазоне длин волн наиболее удобно их выполнять в виде диэлектрической подложки, экранированной с одной стороны и покрытой субволновыми печатными проводниками (патчами) с другой стороны [7, 9]. На рис. 3 показана конструкция такой антенны. Патчи располагаются в квадратных элементарных ячейках со стороной $d$, которая составляет менее четверти длины волны в волноведущей структуре. При таком размере ячейки поверхность является эффективно сплошной. На практике размер $d$ может составлять от $1 / 10$ до $1 / 5$ длины волны. Для реализации тензорного импеданса ячейки должны быть анизотропными, что может быть реализовано различной формой патчей. Например, патчи могут иметь форму эллипсов, кругов с разрезом посередине или любую другую, обеспечивающую несколько геометрических степеней свободы. Например, в случае патчей в форме эллипсов этими степенями свободы являются большая ось эллипса $a$, малая ось эллипса $b$ и угол поворота эллипса $\alpha$ (см. рис. 3 , слева). Кроме этих параметров ячейка рассматриваемой метаповерхности описывается толщиной подложки $h$ и диэлектрической проницаемостью подложки $\varepsilon_{r}$. Параметры подложки выбираются исходя из условий, с одной стороны, достаточно сильного замедления поверхностной волны относительно скорости света, а с другой - отсутствия возбуждения высших типов волн на рабочих частотах. На поверхности с помощью специального фидера (см. рис. 3, справа) возбуждаются цилиндрические поверхностные волны ТМ-типа, которые имеют радиальную поляризацию и при распространении от центра к краю структуры излучаются за счет описанного механизма преобразования поверхностной волны в вытекающую волну. Фидер должен быть спроектирован таким образом, чтобы минимизировать излучение напрямую в свободное пространство и максимум подведенной к антенне энергии трансформировать в энергию поверхностной волны.

Синтез антенны на основе метаповерхности заключается в выборе геометрических параметров патча для каждой ячейки исходя из заданных рабочей частоты, поляризации и направления излучения. Размер всей метаповерхности (апертуры антенны) может быть определен исходя из требуемого коэффициента усиления. В целом процедура синтеза антенны на основе метаповерхности состоит из трех основных этапов: (1) расчета значений тензорного импеданса

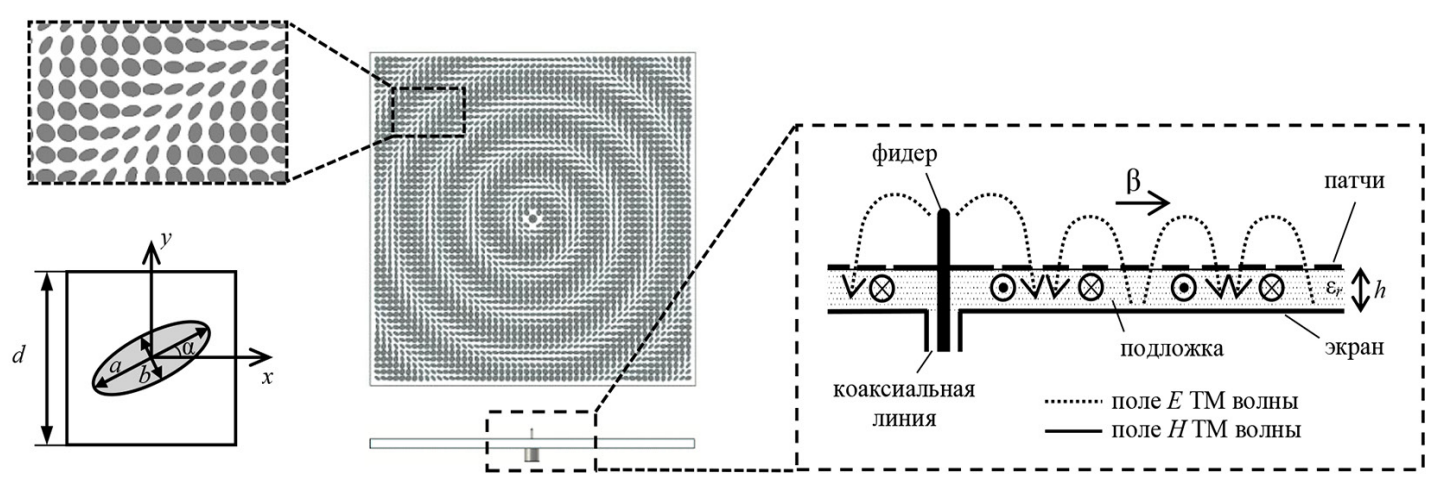

Рис. 3. Конструкция антенны на основе тензорной метаповерхности

Fig. 3. Tensorial metasurface antenna design

$$
-845-
$$




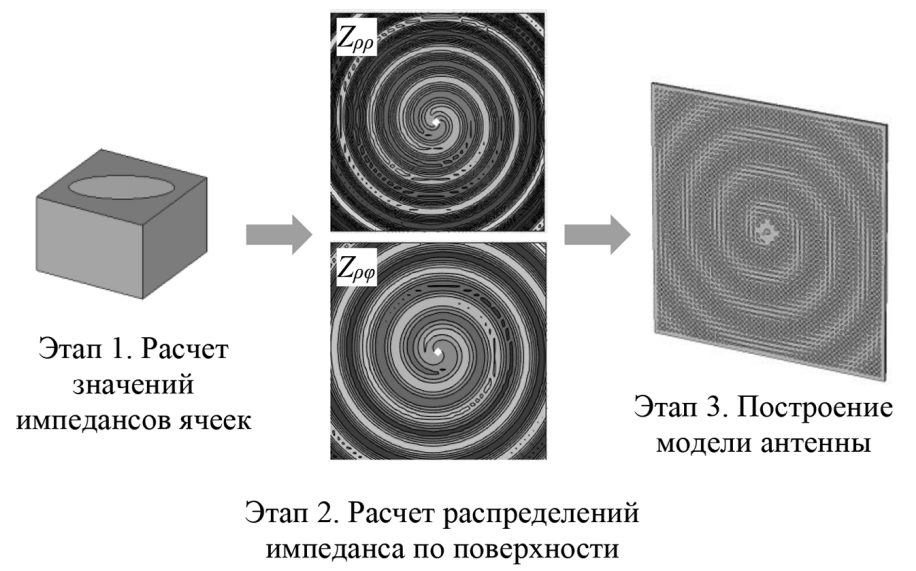

Рис. 4. Последовательность синтеза антенны на основе метаповерхности

Fig. 4. Modulated metasurface antenna synthesis sequence

ячеек, (2) расчета необходимого распределения компонент тензорного импеданса на поверхности, (3) построения антенны (рис. 4).

На первом этапе формируется база данных, ставящая в соответствие каждой форме ячейки определенное значение тензора поверхностного импеданса. Этот этап синтеза по разработанной авторами методике подробно описан в [17].

Второй этап состоит в расчете распределения компонентов тензора импеданса на поверхности, которое необходимо для преобразования цилиндрической ТМ-волны в волну в свободном пространстве с заданными направлением и поляризацией. Этот этап может быть проведен на основе анализа разложения волн на пространственные гармоники и их излучения. Однако математически более простой оказывается методика, основанная на представлении метаповерхности как фазовой голограммы, которая представляет собой записанный в виде изменения поверхностного импеданса (по аналогии с изменением показателя преломления в оптических фазовых голограммах) результат интерференции поверхностной волны (в терминах голографии «опорной» $\left.E_{(r e f)}\right)$ и излучаемой волны (в терминах голографии «объектной» $\left.E_{(o b j)}\right)$. Такой результат интерференции описывается формулой [18]

$$
g=\frac{E_{(o b j)} E_{(r e f)}^{*}}{\left|E_{(r e f)}\right|^{2}} .
$$

В рассматриваемом случае объектная волна является плоской, излучаемой в заданном направлении $\theta_{0}, \varphi_{0}$. Она может быть записана в цилиндрической системе координат, заданной на поверхности антенны как

$$
E_{(o b j)}=A_{(o b j)} e^{-j k_{0}\left(\rho \cos \varphi \sin \theta_{0} \cos \varphi_{0}+\rho \sin \varphi \sin \theta_{0} \sin \varphi_{0}\right)},
$$

где $\rho, \varphi$ - координаты точки на поверхности, а $A_{(o b j)}$ - амплитуда волны.

Опорная волна для рассматриваемого случая цилиндрическая, и если ее возбуждение происходит из точки в центре координат, то на поверхности антенны она будет описываться функцией Ганкеля нулевого порядка: 


$$
E_{(\text {ref })}=A_{(\text {ref })} H_{0}(-\beta \rho)
$$

где $A_{(r e f)}$ - амплитуда волны, $\beta$ - волновое число поверхностной волны.

Поляризация объектной и опорной волн определяется амплитудами и фазами их компонент в ортогональном базисе цилиндрической системы координат:

$$
\begin{aligned}
& E_{(o b j) \rho}=E_{(o b j)} \times e_{(o b j) \rho}, \\
& E_{(o b j) \varphi}=E_{(o b j)} \times e_{(o b j) \varphi}, \\
& E_{(r e f) \rho}=E_{(r e f)} \times e_{(r e f) \rho}, \\
& E_{(r e f) \varphi}=E_{(r e f)} \times e_{(r e f) \varphi},
\end{aligned}
$$

где $e_{(o b j) p} e_{(o b j) \varphi}-$ комплексные направляющие косинусы для объектной волны, $e_{(r e f) \rho} e_{(r e f) \varphi}-$ комплексные направляющие косинусы для опорной волны.

Для поверхностной цилиндрической ТМ-волны, возбуждаемой в начале координат, направляющие косинусы определяются как

$$
e_{(r e f) \rho}=1, e_{(r e f) \varphi}=0
$$

так как на поверхности присутствует только радиальная продольная компонента электрического поля.

Для излучаемой плоской волны комплексные направляющие косинусы могут быть заданы произвольно, в зависимости от требуемой поляризации. Например, для правой круговой поляризации они будут равны

$$
\begin{aligned}
& e_{(o b j) \rho}=\sqrt{0.5}\left(\cos \varphi+e^{j \frac{\pi}{2}} \sin \varphi\right) \\
& e_{(o b j) \varphi}=\sqrt{0.5}\left(\sin \varphi-e^{j \frac{\pi}{2}} \cos \varphi\right) .
\end{aligned}
$$

В голографической методике синтеза компоненты тензора импеданса модулируются относительно своих средних значений по закону (9):

$$
\begin{aligned}
& Z_{\rho \rho}=\bar{Z}_{\rho \rho}+\frac{\Delta Z_{\rho \rho}}{2} \times \operatorname{Re}\left(\frac{E_{(o b j) \rho} E_{(r e f) \rho}^{*}}{\left|E_{(r e f)}\right|^{2}}\right), \\
& Z_{\varphi \varphi}=\bar{Z}_{\varphi \varphi}+\frac{\Delta Z_{\varphi \varphi}}{2} \times \operatorname{Re}\left(\frac{E_{(o b j) \varphi} E_{(r e f) \varphi}^{*}}{\left|E_{(r e f)}\right|^{2}}\right), \\
& Z_{\rho \varphi}=\bar{Z}_{\rho \varphi}+\frac{\Delta Z_{\rho \varphi}}{2} \times \operatorname{Re}\left(\frac{E_{(o b j) \rho} E_{(r e f) \varphi}^{*}}{\left|E_{(r e f)}\right|^{2}}\right), \\
& Z_{\varphi \rho}=\bar{Z}_{\varphi \rho}+\frac{\Delta Z_{\varphi \rho}}{2} \times \operatorname{Re}\left(\frac{E_{(o b j) \varphi} E_{(r e f) \rho}^{*}}{\left|E_{(r e f)}\right|^{2}}\right), \\
& -847-
\end{aligned}
$$


где $\bar{Z}_{\rho \rho}, \bar{Z}_{\varphi \varphi}, \bar{Z}_{\rho \varphi}, \bar{Z}_{\varphi \rho}$ - средние значения компонента импеданса, реализуемого ячейками, $\Delta Z_{\rho \rho}$, $\Delta Z_{\varphi \varphi}, \Delta Z_{\rho \varphi}, \Delta Z_{\varphi \rho}$ - диапазон значений соответствующего компонента импеданса. Эти значения определяются из базы данных импедансов ячеек, полученной на первом этапе синтеза.

На рис. 4 в центре приведены распределения только двух компонент тензора $-Z_{\rho \rho}$ и $Z_{\rho \varphi}$. Именно эти два компонента определяют свойства поверхности, так как $Z_{\varphi \varphi}=$ const в силу того, что $e_{(r e f) \varphi}=0$, а $Z_{\varphi \rho}=Z_{\rho \varphi}$ в силу того, что тензор импеданса является диагональным для взаимных сред.

Третий этап синтеза (см. рис. 4) заключается в подборе параметров патча для каждой ячейки поверхности и построении антенны. Он может быть легко осуществлен, если известно необходимое распределение компонентов тензора импеданса на поверхности, получаемое на втором этапе, и значения тензора импеданса, реализуемые каждой формой патча, получаемые на первом этапе. Строгая реализация распределений всех компонент тензора одновременно, как правило, невозможна, поэтому на третьем этапе синтеза необходимо подобрать для каждой точки поверхности ячейку, максимально близко удовлетворяющую одновременно всем независимым компонентам тензора. На практике это оказывается возможным с достаточной точностью, если форма патча допускает по крайней мере три геометрических степени свободы.

Экспериментальные результаты. Для экспериментальной проверки описанной методики синтеза антенн на основе тензорных метаповерхностей были синтезированы и изготовлены три антенны Ки-диапазона. Все они имели одинаковые габариты 380х380 мм, центральную частоту 12 ГГц (длина волны $\lambda_{0}=25$ мм) и были изготовлены на материале FR4 $\left(\varepsilon_{r}=4.5\right.$, $\left.\tan \delta_{\varepsilon}=0.022\right)$ толщиной 2 мм по стандартной технологии изготовления печатных плат. Апертура антенн имела форму восьмигранника. Антенна № 1 имела правую круговую поляризацию и направление главного лепестка по нормали к поверхности, антенна № 2 - такое же направление главного лепестка, но линейную поляризацию, а антенна № 3 - левую круговую поляризацию и направление главного лепестка $45^{\circ}$ от нормали к поверхности.

Была использована форма патча элементарной ячейки метаповерхности в виде эллипса. Размер элементарной ячейки составлял $3 \times 3$ мм, или $0.12 \times 0.12 \lambda_{0}$. Поверхность каждой антенны состояла приблизительно из 14200 ячеек, для каждой из которых с помощью алгоритма синтеза были подобраны параметры $a, b$ и $\alpha$. Для возбуждения поверхностной волны ТМ-типа был использован фидер в виде штыря с диском, аналогичный изображенному на рис. 3. Диаметр диска фидера на верхней стороне подложки составлял 2 мм, а высота штыря 5.5 мм. Для подключения к фидеру использовался SMA-разъем.

На рис. $5 a$ показана фотография антенны № 1 в безэховой камере, а на рис. 56 - поверхность антенны. Антенны № 2 и № 3 выглядели аналогично, за исключением рисунка метаповерхности.

Было проведено измерение характеристик направленности изготовленных антенн методом сканирования ближнего поля. Измеренные характеристики сравнивались с результатами численного моделирования методом конечных элементов в программе CST Studio Suite. На рис. 6 показаны измеренные диаграммы направленности антенны № 1 на частоте 12 ГГц для правой круговой поляризации в двух сечениях - по азимуту и по элевации. Также для сравнения приведены результаты расчета. Как видно, в области главного лепестка рассчитанные и измеренные диаграммы направленности имеют хорошее совпадение. Направление главно- 


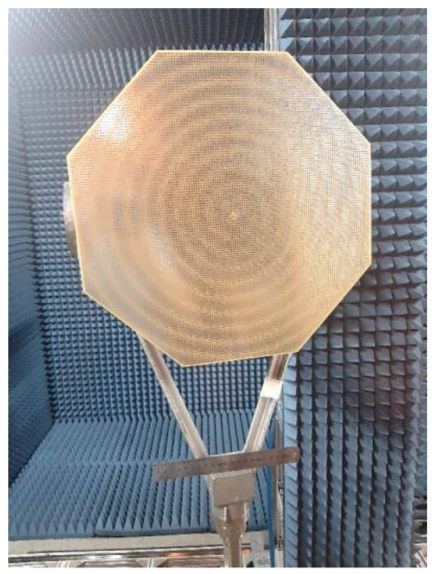

(a)

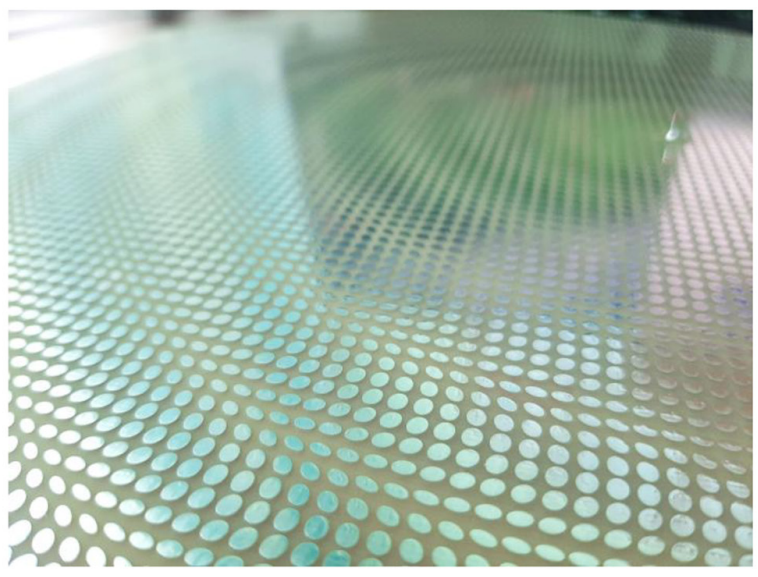

(б)

Рис. 5. Антенна № 1: $a$ - антенна в безэховой камере; $\sigma$ - поверхность антенны

Fig. 5. Antenna \#1: $a$ - antenna in anechoic chamber, $b$ - antenna surface

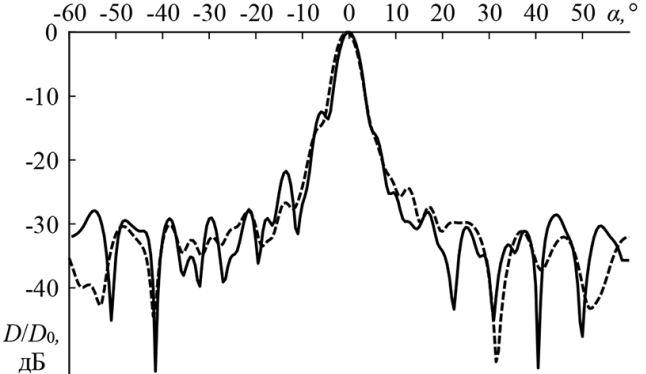

(a)

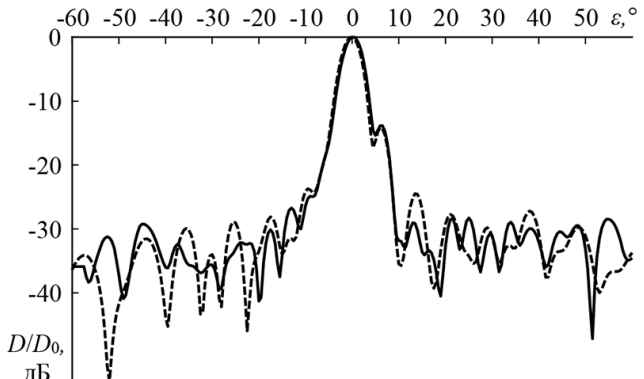

(б)

Рис. 6. Диаграммы направленности антенны № 1 на частоте 12 ГГц: $a$ - сечение по азимуту; $\sigma$ - сечение по элевации; пунктир - расчет; сплошные линии - эксперимент

Fig. 6. Antenna \#1 radiation patterns at $12 \mathrm{GHz}: a$ - azimuth cut; $b$ - elevation cut; dashed lines - simulation; solid lines - measurement

го лепестка и поляризация совпадают с заданными при синтезе, что подтверждает корректность использованной методики. Ширина главного лепестка по уровню минус 3 дБ составила $4^{\circ}$ в обеих плоскостях, а уровень кросс-поляризации в направлении максимума излучения составил минус 26 дБ.

На рис. 7 дано сравнение измеренного и рассчитанного коэффициентов отражения от входа антенны. В рабочем диапазоне частот он не превышает минус 9 дБ, а вклад потерь на рассогласование в общие потери в антенне не превышает 0.5 дБ. На рис. 8 изображены частотные зависимости коэффициента усиления (КУ) и коэффициента направленного действия (КНД) антенны № 1. Здесь также наблюдается хорошее совпадение с результатами расчета. Максимальный КНД составил 31 дБи, что соответствует апертурной эффективности антенны 52 \%, а максимальный КУ составил 26 дБи, что соответствует полной эффективности антенны 17 \%. Как видно, тепловые потери в антенне (разница между КНД и КУ) значительны, около 5 дБ. Это вызвано высоким значением тангенса угла диэлектрических потерь использованно- 


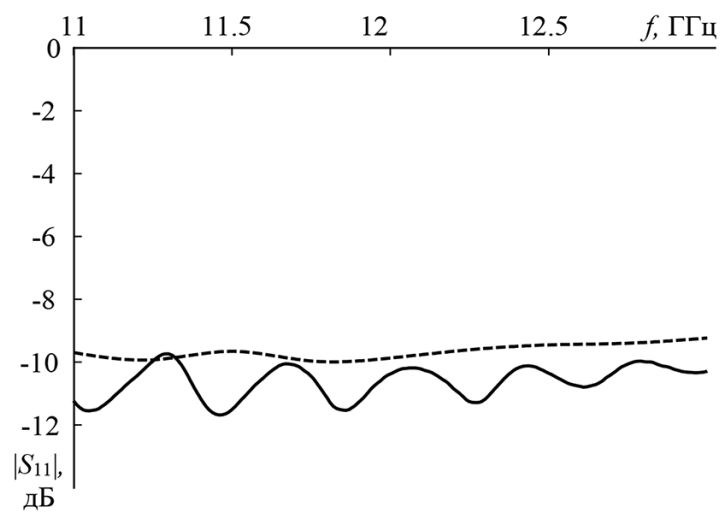

Рис. 7. Коэффициент отражения антенны № 1 (пунктир - расчет, сплошные линии - эксперимент)

Fig. 7. Antenna \#1 reflection coefficient (dashed lines simulation, solid lines - measurement)

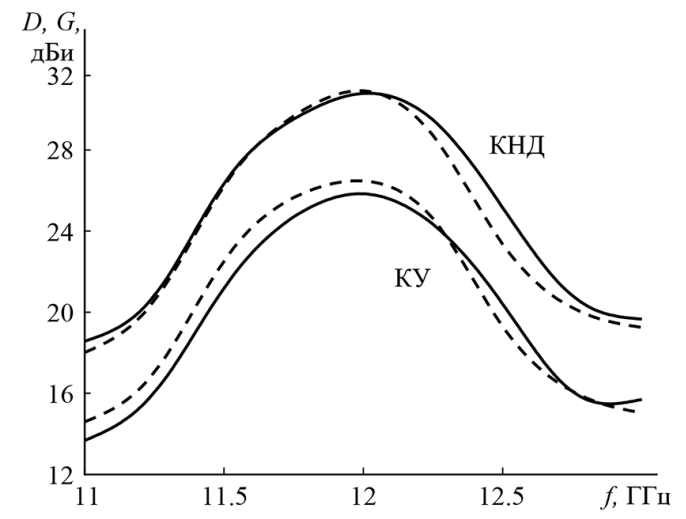

Рис. 8. Коэффициент направленного действия и коэффициент усиления антенны № 1 (пунктир расчет, сплошные линии - эксперимент)

Fig. 8. Antenna \#1 directivity and gain (dashed lines simulation, solid lines - measurement)

го материала подложки (FR-4). При применении в качестве подложки специализированных CBЧ-материалов с низким тангенсом угла диэлектрических потерь, например Taconic RF-45 $\left(\varepsilon_{r}=4.5, \tan \delta_{\varepsilon}=0.0035\right)$, тепловые потери могут быть уменьшены до 1 дБ, а полная эффективность антенны увеличена до 40 \%. Ширина полосы рабочих частот антенны по критерию снижения КУ на 3 дБ составила $6 \%$.

Аналогичным образом были измерены характеристики антенн № 2 и № 3. Результаты проведенных измерений всех трех антенн сведены в таблицу. Во всех случаях они хорошо соответствуют результатам численного моделирования и параметрам, заданным при синтезе. Также в таблице для наглядности приведены распределения компонента тензора $Z_{\rho \rho}$ по поверхности антенны, полученные в ходе синтеза. Значения эффективности антенны № 3 , имеющей направление главного лепестка $45^{\circ}$, были рассчитаны с учетом снижения рабочей площади антенны по закону $\cos \theta$ при отклонении направления излучения.

Заключение. В результате работы экспериментально подтверждено, что предложенная методика синтеза антенн на основе тензорных метаповерхностей позволяет получать антенны с заданными направлением излучения и поляризацией, которые обладают апертурной эффективностью до $50 \%$, шириной полосы рабочих частот до 8 \% и уровнем кросс-поляризации ниже минус 20 дБ. Результаты измерений антенн хорошо согласуются с электродинамическим расчетом и заданными при синтезе параметрами.

На основе проведенных исследований можно сделать вывод, что АМП обладают такими достоинствами, как низкий профиль, технологичность производства, достаточно высокая эффективность и возможность получения различных форм диаграммы направленности только за счет изменения рисунка метаповерхности. К их недостаткам можно отнести сравнительно узкую полосу рабочих частот. Тем не менее, на взгляд авторов, такие антенны являются перспективными для различных применений, например, в спутниковой и мобильной связи.

Следует выделить несколько особенностей АМП. Во-первых, способ распределения мощности по апертуре антенны за счет бегущей поверхностной волны существенно более прост, 
Таблица. Характеристики макетов антенн

Table. Manufactured antennas performance

\begin{tabular}{|l|c|c|c|}
\hline \multicolumn{1}{|c|}{ Характеристика } & Антенна № 1 \\
\hline Распределение компонента тензора $Z_{\rho \rho}$ & & \\
\hline
\end{tabular}

чем параллельные или последовательные схемы деления, применяемые в антенных решетках, особенно при больших электрических размерах антенны, что позволяет получать меньшие тепловые потери и потери на рассогласование при прочих равных условиях. Во-вторых, электрический размер апертуры АМП ограничен снизу, так как на апертуре малого размера энергия поверхностной волны не излучается полностью даже при максимальной глубине модуляции импеданса поверхности. Часть энергии, дошедшая до края антенны, не участвует в формировании заданной диаграммы направленности, что снижает эффективность антенны. В-третьих, полная эффективность АМП зависит от большого числа факторов: согласования внешнего СВЧ тракта с фидером, эффективности возбуждения фидером поверхностной волны, равномерности создаваемого вытекающими волнами амплитудного и фазового распределений, долей энергии поверхностной волны, дошедшей до края апертуры, потерями в металле патчей и диэлектрике подложки. Все эти параметры необходимо тщательно оптимизировать для получения полной эффективности 60-70 \%, которая может считаться достаточной для практических применений.

В дальнейшей работе планируется совершенствование разработанной методики синтеза с целью увеличения эффективности получаемых АМП, а также синтеза АМП с произвольной формой диаграммы направленности.

$$
\text { - } 851-
$$




\section{Благодарности / Acknowledgements}

Исследование выполнено при финансовой поддержке РФФИ, Правительства Красноярского края и Красноярского краевого фонда науки в рамках научного проекта 20-47243002.

The research was carried out with the financial support of the RFBR, the Government of the Krasnoyarsk Territory and the Krasnoyarsk Regional Science Foundation within the framework of the scientific project 20-47-243002.

\section{Список литературы / References}

[1] Glybovski S.B., Tretyakov S.A., Belov P.A., Kivshar Y.S., Simovski C.R. Metasurfaces: From microwaves to visible. Physics Reports, 2016, 634, 1-72.

[2] Jackson D.R., Caloz C., Itoh T. Leaky-Wave Antennas. Proceedings of the IEEE, 2012, 100(7), 2194-2206.

[3] Oliner A., Hessel A. Guided waves on sinusoidally-modulated reactance surfaces. IRE Transactions on Antennas and Propagation, 1959, 7(5), 201-208.

[4] Конторович М.И. Усредненные граничные условия на поверхности сетки с квадратными ячейками. Радиотехника и электроника, 1963, 8, 1506-1515. [Kontorovich M.I. Average boundary conditions on the surface of a grid with square cells. Radiotehnika i elektronika, 1963, 8, 1506-1515 (in Russisn)].

[5] Чаплин А.Ф. Возбуждение импедансной плоскости с периодическими сосредоточенными неоднородностями. Радиотехника и электроника, 1986, 5, 11-19. [Chaplin A. F. Excitation of an impedance plane with periodic lumped inhomogeneities, Radiotehnika i elektronika, 1986, 5, 11-19 (in Russian)].

[6] Fong B.H., Colburn J.S., Ottusch J.J., Visher J.L., Sievenpiper D.F. Scalar and Tensor Holographic Artificial Impedance Surface. IEEE Transactions on Antennas and Propagation, 2010, 58(10), 3212-3221.

[7] Minatti G., Caminita F., Martini E., Sabbadini M., Maci S. Synthesis of ModulatedMetasurface Antennas With Amplitude, Phase, and Polarization Control. IEEE Transactions on Antennas and Propagation, 2016, 64 (9), 3907-3919.

[8] Minatti G., Caminita F., Martini E., Maci S. Flat Optics for Leaky-Waves on Modulated Metasurfaces: Adiabatic Floquet-Wave Analysis. IEEE Transactions on Antennas and Propagation, 2016, 64(9), 3896-3906.

[9] Teniou M., Roussel H., Serhir M., Capet N., Piau G.-P., Casaletti M. Tensorial metasurface antennas radiating polarized beams based on aperture field implementation. International Journal of Microwave and Wireless Technologies, 2017, 10(2), 161-168.

[10]Tcvetkova S.N., Martini E., Tretyakov S.A., Maci S. Perfect Conversion of a TM Surface Wave Into a TM Leaky Wave by an Isotropic Periodic Metasurface Printed on a Grounded Dielectric Slab. IEEE Transactions on Antennas and Propagation, 2020, 68(8), 6145-6153.

[11] Minatti G., Martini E., Maci S. Efficiency of Metasurface Antennas. IEEE Transactions on Antennas and Propagation, 2017, 65(4), 1532-1541.

[12]Faenzi M., Minatti G., González-Ovejero D. Metasurface Antennas: New Models, Applications and Realizations. Scientific Reports, 2019, 9(1), 10178. 
[13]Kim S., Shrekenhamer D., Will J., Awadallah R., Miragliotta J. High impedance holographic metasurfaces for conformal and high gain antenna applications. 15th IEEE Annual Consumer Communications \& Networking Conference (CCNC), 2018, 1-4.

[14]Deng R., Di B., Zhang H., Tan Y. and Song L. Reconfigurable Holographic Surface: Holographic Beamforming for Metasurface-Aided Wireless Communications. IEEE Transactions on Vehicular Technology, 2021, 70(6), 6255-6259.

[15]Yurduseven O., Podilchak S., Khalily M. Towards Holographic Beam-Forming Metasurface Technology for Next Generation CubeSats. IEEE International Conference on UK-China Emerging Technologies (UCET), 2020, 1-4.

[16]González-Ovejero D., Chahat N., Sauleau R., Chattopadhyay G., Maci S., Ettorre M. Additive Manufactured Metal-Only Modulated Metasurface Antennas. IEEE Transactions on Antennas and Propagation, 2018, 66(11), 6106-6114.

[17]Lemberg K.V., Kosmynin A. N., Alexandrin A. M., Grushevsky E. O.,Podshivalov I. V. Method of Anisotropic Metasurface Unit Cell Surface Impedance Calculation. Proceedings of Radiation and Scattering of Electromagnetic Waves 2021 Conference, 2021, 1-3.

[18]Hariharan P. Optical Holography: Principles, Techniques and Applications. Cambridge, U.K.: Cambridge Univ. Press, 1996. P. 11-13. 